\title{
Instabilities driven by higher-order modes in a radio frequency system with a passive higher-harmonic cavity
}

\author{
R. A. Bosch \\ Synchrotron Radiation Center, University of Wisconsin-Madison, 3731 Schneider Drive, Stoughton, Wisconsin 53589 USA
}

(Received 10 November 2004; published 4 August 2005)

\begin{abstract}
The effect of a higher-order mode upon longitudinal beam stability in an electron storage ring is modeled analytically and with simulations. Narrow band parasitic modes and broadband impedance are considered for the Aladdin and MAX-II electron storage rings. The simulations confirm that a passive harmonic cavity strongly suppresses parasitic coupled-bunch instabilities, in agreement with the analytic model. In the long-bunch regime where the bunch length exceeds the vacuum pipe radius, analytic modeling and simulations indicate that a harmonic cavity also suppresses the microwave instability. In the short-bunch regime where the bunch length is smaller than the vacuum pipe radius, analytic modeling and simulations indicate that tuning in a harmonic cavity may worsen the microwave instability.
\end{abstract}

DOI: 10.1103/PhysRevSTAB.8.084401

PACS numbers: 29.27.Bd, 29.20.Dh, 41.75.Ht

\section{INTRODUCTION}

A passive harmonic radio-frequency (rf) cavity may be utilized to lengthen the bunch and suppress longitudinal parasitic coupled-bunch instabilities [1-5]. When all rf buckets are filled equally (so that transient beam loading $[6,7]$ may be neglected), the harmonic-cavity voltage required to suppress parasitic coupled-bunch instabilities may be predicted analytically [8]. For harmonic-cavity operation at Aladdin (in high- and low- emittance lattices) and the UVSOR electron storage ring, the analytic predictions are in approximate agreement with experiment [810]. Consideration of the negative-mass instability of a coasting beam provides an approximate analytic criterion for predicting the microwave instability [11] that is similar to that of the ZAP code [12].

To simulate the suppression of instabilities by a harmonic cavity [13-16], we include a higher-order mode (HOM) in a simulation code developed to study Robinson instabilities [8]. To speed computations, wake fields are represented by in-phase and quadrature components [17].

For the Aladdin $800-\mathrm{MeV}$ electron storage ring, which employs a $50-\mathrm{MHz}$ fundamental rf cavity and a $200-\mathrm{MHz}$ harmonic cavity, we study two tunings of the ring's quadrupole and sextupole magnets. The first tuning is the "base lattice," while the second tuning is a low-emittance lattice whose momentum compaction is much lower. We also study the MAX-II $1.5-\mathrm{GeV}$ electron storage ring with its new $100-\mathrm{MHz}$ rf system with a 500-MHz harmonic cavity. For all three cases, parasitic coupled-bunch instabilities in simulations of a typical HOM are strongly suppressed by tuning in the harmonic cavity, in agreement with the analytic modeling. At Aladdin, parasitic coupled-bunch instabilities have also been studied experimentally [8], confirming the suppression of instability.

For Aladdin operation in either the base-lattice or lowemittance lattice, the natural bunch length exceeds the vacuum-chamber radius. In this long-bunch regime of the microwave instability, analytic modeling and simulations were performed for the estimated reduced longitudinal broadband impedance of the vacuum chamber, $\left|Z_{p} / p\right|=$ $5.7 \Omega$ [18]. Simulations of the bunch length for singlebunch operation of the low-emittance lattice are in approximate agreement with experiment, suggesting that the value of $5.7 \Omega$ is reasonable. In this long-bunch regime of the microwave instability, analytic modeling and simulations indicate that tuning in the harmonic cavity suppresses microwave instability. The microwave instability thresholds in experiments with and without bunch lengthening by the harmonic cavity are in good agreement with simulations, further confirming the suppression of the microwave instability.

For MAX-II, we also study microwave instability with broadband reduced impedance of $5.7 \Omega$. Here, the natural bunch length is smaller than the vacuum chamber radius. In this short-bunch regime of the microwave instability, analytic modeling and simulations predict that the microwave instability is worsened as the harmonic cavity is initially tuned in. When the harmonic cavity is tuned in further so that the bunch length is in the long-bunch regime of the microwave instability, additional bunch lengthening is expected to suppress the microwave instability. Consequently, the microwave instability is not observed in simulations of MAX-II operation with optimally lengthened bunches.

\section{INSTABILITY MODELING}

We consider the parasitic coupled-bunch instability and the microwave instability for the Aladdin ring operated in base-lattice and low-emittance tunings with a 50-MHz/ 200-MHz rf system [19], and the MAX-II ring operated with the new $100-\mathrm{MHz} / 500-\mathrm{MHz}$ rf system [20,21]. Analytic predictions are compared with multiparticle simulations for a variety of ring currents and harmoniccavity resonant frequencies. The analytic predictions and simulations are complimentary, in which the analytic 
model describes the mechanism of instability, while the energy spread in simulations shows the severity. Comparisons with Aladdin experiments indicate that simulations predict microwave instability thresholds more accurately than the analytic model.

The simulations include radiation damping, random energy fluctuations from synchrotron radiation, excitation of wake fields, and the effect of the wake fields upon the macroparticles. We include wake fields in the dominant modes of the fundamental and harmonic cavities, and in a HOM. For each turn around the machine, the energy offset of the $m$ th macroparticle $\varepsilon_{m}$ is modified by

$$
\begin{aligned}
\Delta \varepsilon_{m}= & \left(-2 T_{\mathrm{o}} / \tau_{L}\right) \varepsilon_{m}+2 \sigma_{E_{\text {nat }}}\left(T_{\mathrm{o}} / \tau_{L}\right)^{1 / 2} R_{m n}-e V_{s} \\
& +e V\left(t_{m}\right)
\end{aligned}
$$

where $T_{\mathrm{o}}$ is the recirculation time, $\tau_{L}$ is the longitudinal radiation-damping time, $\sigma_{E_{\text {nat }}}$ is the natural energy spread, $R_{m n}$ is a random number with zero mean and unity standard deviation, $e>0$ is the magnitude of the electron charge, $V_{s}$ is the synchronous voltage, $t_{m}$ is the macroparticle arrival time, and $V\left(t_{m}\right)$ is the rf voltage from the dominant modes of the fundamental and harmonic cavities and the HOM. The macroparticle time offset is modified each turn by

$$
\Delta t_{m}=\alpha T_{\mathrm{o}} \varepsilon_{m} / E,
$$

where $\alpha$ is the momentum compaction and $E$ is the ring energy. Excitation and/or damping of Robinson oscillations results from the macroparticle wakefields in the dominant modes of the fundamental and harmonic rf cavities [8]. This contrasts with simulation codes that neglect these wakefields and compensate with a dipole Robinsondamping term [22-24].

To speed up the simulations, the rf voltages (which include the macroparticle wakes) are described using "in-phase" and "quadrature" components [17], as discussed in the appendix. The equations for a HOM do not assume that the HOM has a high quality factor. We use the same code for simulating parasitic coupled-bunch instability from a high- $Q$ HOM and for simulating microwave instability from low- $Q$ broadband impedance.

The harmonic-cavity resonant frequency is characterized by the tuning angle $\phi_{2}$, defined by $\tan \phi_{2} \equiv$ $2 Q_{2}\left(\nu \omega_{g}-\omega_{2}\right) / \omega_{2}$, where $\nu, Q_{2}$, and $\omega_{2}$ are the harmonic number, quality factor, and resonant angular frequency of the harmonic cavity's dominant mode, while $\omega_{g}$ is the rf angular frequency. To ensure that sufficiently many macroparticles are simulated, we first study the Aladdin ring in low-emittance tuning with no harmonic cavity, which is equivalent to $\phi_{2}=-90^{\circ}$.

\section{A. Operation without a harmonic cavity}

A HOM with quality factor $Q_{3}=3000$, resonant impedance $R_{3}=10 \mathrm{k} \Omega$, and resonant angular frequency $\omega_{3} \approx 2 \pi \times 10^{9}$ radians/s represents a typical parasitic mode in a cavity with HOM damping [21]. To approximately obtain worst-case instability growth with longitudinal mode number different from 0,1 , or -1 , the resonant frequency of the higher-order mode $\left(\omega_{3}\right)$ is adjusted to equal a multiple of the ring's revolution frequency. Parameters are given in Table I.

TABLE I. Parameters for the Aladdin base and low-emittance lattices, and MAX-II with the new rf system. The shunt impedance

\begin{tabular}{|c|c|c|c|c|}
\hline Parameter & Symbol & Aladdin base lattice & Aladdin low-emittance lattice & MAX-II \\
\hline Ring energy & $E$ & $800 \mathrm{MeV}$ & $800 \mathrm{MeV}$ & $1.5 \mathrm{GeV}$ \\
\hline Natural relative energy spread & $\sigma_{E_{\text {nat }}} / E$ & $4.8 \times 10^{-4}$ & $4.8 \times 10^{-4}$ & $7 \times 10^{-4}$ \\
\hline Synchronous voltage & $V_{s}$ & $17.4 \mathrm{kV}$ & $17.4 \mathrm{kV}$ & $140 \mathrm{kV}$ \\
\hline Recirculation time & $T_{\mathrm{o}}$ & $2.96 \times 10^{-7} \mathrm{~s}$ & $2.96 \times 10^{-7} \mathrm{~s}$ & $3 \times 10^{-7} \mathrm{~s}$ \\
\hline Number of bunches & $M$ & 15 & 15 & 30 \\
\hline Fundamental cavity shunt impedance & $R_{1}{ }^{\mathrm{o}}$ (unloaded) & $0.5 \mathrm{M} \Omega$ & $0.5 \mathrm{M} \Omega$ & $4.8 \mathrm{M} \Omega$ \\
\hline Fundamental cavity quality factor & $Q_{1}{ }^{\mathrm{o}}$ (unloaded) & 8000 & 8000 & 19000 \\
\hline Fundamental cavity coupling coefficient & $\beta_{1}$ & 11 & 11 & 3 \\
\hline Harmonic-cavity harmonic number & $\nu$ & 4 & 4 & 5 \\
\hline Harmonic-cavity shunt impedance & $R_{2}{ }^{\mathrm{o}}$ (unloaded) & $1.24 \mathrm{M} \Omega$ & $1.24 \mathrm{M} \Omega$ & $1.7 \mathrm{M} \Omega$ \\
\hline Harmonic-cavity quality factor & $Q_{2}^{\circ}$ (unloaded $)$ & 20250 & 20250 & 24000 \\
\hline Harmonic-cavity coupling coefficient & $\beta_{2}$ & 0 & 1.5 & 0 \\
\hline HOM resonant angular frequency & $\omega_{3}$ & $6.28319 \times 10^{9} \mathrm{rad} / \mathrm{s}$ & $6.28319 \times 10^{9} \mathrm{rad} / \mathrm{s}$ & $6.220353 \times 10^{9} \mathrm{rad} / \mathrm{s}$ \\
\hline HOM resonant impedance & $R_{3}$ & $10 \mathrm{k} \Omega$ & $10 \mathrm{k} \Omega$ & $10 \mathrm{k} \Omega$ \\
\hline HOM quality factor & $Q_{3}$ & 3000 & 3000 & 3000 \\
\hline Fundamental cavity maximum voltage & $V_{T 1}$ & $90 \mathrm{kV}$ & $50 \mathrm{kV}$ & $450 \mathrm{kV}$ \\
\hline Fundamental cavity loadangle & $\theta_{\mathrm{v} 1}$ & 0 degrees & 0 degrees & 0 degrees \\
\hline Momentum compaction & $\alpha$ & 0.0335 & 0.006 & 0.004 \\
\hline Radiation-damping time & $\tau_{L}$ & $13.8 \mathrm{~ms}$ & $13.5 \mathrm{~ms}$ & $3.2 \mathrm{~ms}$ \\
\hline
\end{tabular}
value equals the resistance of the equivalent RLC circuit, and is equal to one-half of the "linac" definition of shunt impedance. The HOM parameters describe a parasitic HOM. 
For simulations of $1 \times 10^{6}$ turns with a parasitic HOM, the relative energy spread and the rms macroparticle arrival time (with respect to the synchronous phase) were averaged over the final 500000 turns. Since all rf modes in this case have a high quality factor, beam loading and instabilities result from all macroparticles in the simulation. Figure 1 shows the dependence of energy spread and rms arrival time upon the bunch current when all $15 \mathrm{rf}$ buckets are filled, for simulations utilizing different numbers of macroparticles. Instability occurs for bunch currents exceeding $0.25 \mathrm{~mA}$, i.e., ring currents exceeding $3.75 \mathrm{~mA}$. When simulating at least 1000 macroparticles, the results have little dependence upon the number of macroparticles.

For the Aladdin low-emittance lattice, the microwave instability may be driven by the reduced longitudinal broadband impedance of the vacuum chamber, estimated to be $\left|Z_{p} / p\right| \approx 5.7 \Omega$. The estimated cutoff angular frequency is $\omega_{c}=c / b=8 \times 10^{9} \mathrm{rad} / \mathrm{s}$ [18], where $c$ is the speed of light and $b=36 \mathrm{~mm}$ is the radius of the round vacuum-chamber pipe. We model the broadband impedance by a HOM with $\omega_{3}=8 \times 10^{9} \mathrm{rad} / \mathrm{s}, Q_{3}=1$ and $R_{3}=2148 \Omega$. The effect of broadband impedance upon a bunch of rms length $\sigma_{t}$ (measured in units of time) is different in the long-bunch regime (where $\omega_{3} \sigma_{t}>1$ )
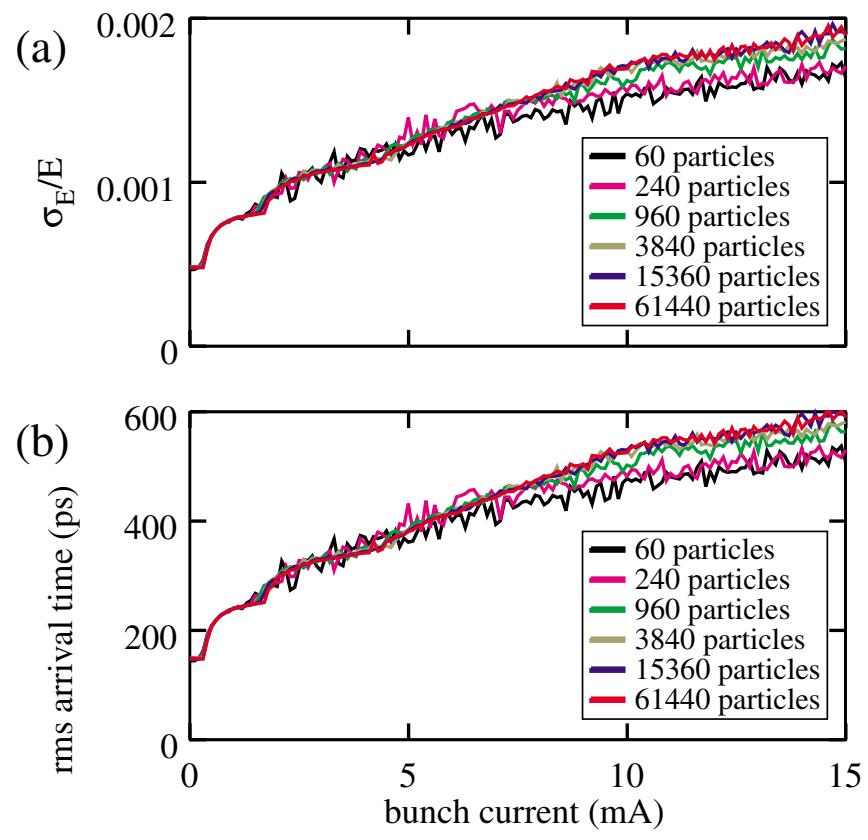

FIG. 1. (Color) The relative energy spread and rms macroparticle arrival time of the Aladdin low-emittance lattice are shown for operation with all 15 rf buckets filled, with the harmonic cavity detuned. Simulated values for a parasitic impedance with quality factor $Q_{3}=3000$, resonant impedance $R_{3}=10 \mathrm{k} \Omega$, and resonant angular frequency $\omega_{3}=6.28319 \times 10^{9} \mathrm{rad} / \mathrm{s}$ are plotted for simulations using different numbers of macroparticles. The simulated values are averages over the final 500000 turns of $1 \times 10^{6}$-turn simulations with harmonic-cavity tuning angle of $-90^{\circ}$. than in the short-bunch regime (where $\omega_{3} \sigma_{t}<1$ ) [13]. For our broadband impedance model in which $\omega_{3}=\omega_{c}$, the long-bunch regime describes bunch lengths exceeding the vacuum-chamber radius, while the short-bunch regime describes bunch lengths smaller than the vacuum-chamber radius.

Instabilities driven by broadband impedance are expected to primarily involve the interaction of the macroparticles in a single bunch. Figure 2 shows the relative energy spread and bunch length plotted versus current for single-bunch simulations with different numbers of macroparticles. When the number of macroparticles is at least 1000 , the results are nearly independent of the number of macroparticles. When the bunch current exceeds $5 \mathrm{~mA}$, the relative energy spread $\sigma_{E} / E$ and bunch length $\sigma_{t}$ (measured in units of time) are increased by the microwave instability. For lower bunch currents, potential-well distortion lengthens the bunch, since the broadband impedance is inductive to the bunch for $\omega_{3} \sigma_{t}>1$. In Fig. 2(b), the simulated bunch length is compared with measurements. The approximate agreement suggests that our estimated broadband impedance of $5.7 \Omega$ is reasonable.

Simulations displaying unstable behavior (such as oscillations of the bunch positions or length) have energy spreads exceeding the natural value [8]. Energy spreads
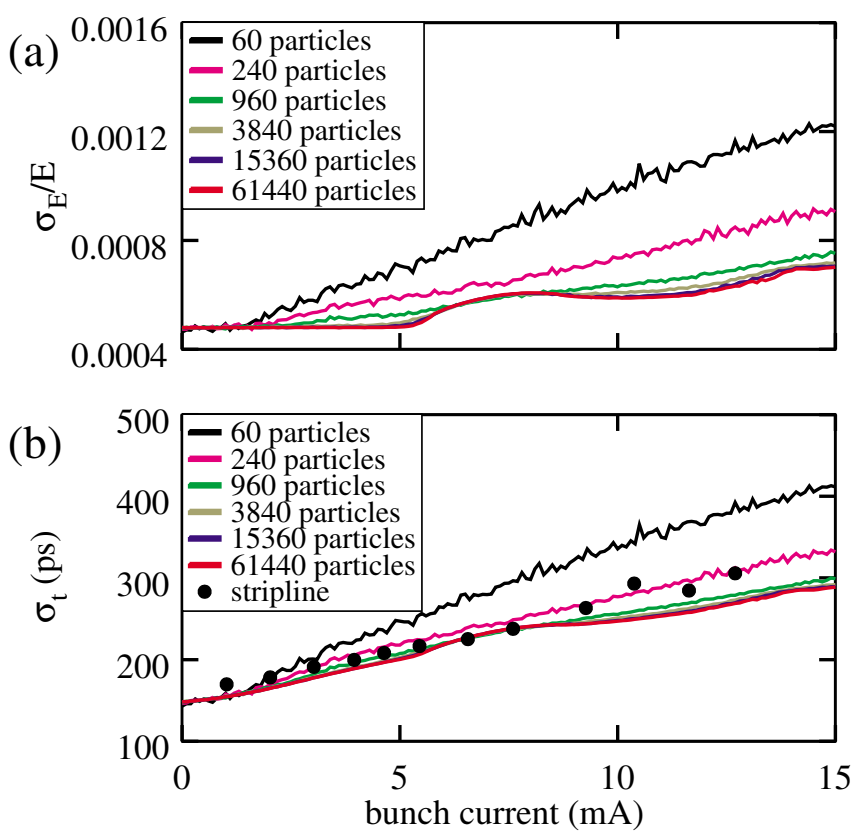

FIG. 2. (Color) The relative energy spread and rms bunch length of the Aladdin low-emittance lattice are shown for single-bunch operation with the harmonic cavity detuned. Simulated values for a broadband impedance of $\left|Z_{p} / p\right|=5.7 \Omega$ are plotted for simulations using different numbers of macroparticles. The simulated values are averages over the final 500000 turns of $1 \times$ $10^{6}$-turn simulations with harmonic-cavity tuning angle of $-90^{\circ}$. Measured bunch lengths were obtained from the voltage induced upon a $0.365-\mathrm{m}$ strip line. 
exceeding the natural value by $>10 \%$ may be taken as an indication of observable unstable behavior. According to Figs. 1 and 2, the simulated energy spread may be determined within $10 \%$ provided that at least 1000 macroparticles are used to model high- $Q$ modes, and at least 1000 macroparticles per bunch are used to model broadband impedance. Simulations with at least 1000 macroparticles per bunch are expected to model broadband impedance in addition to beam loading and instabilities from the dominant high- $Q$ modes of the fundamental and harmonic cavities.

To evaluate stability for ring operation, we perform 500000 -turn simulations for 15 values of the ring current and 30 values of the harmonic-cavity tuning angle, with all rf buckets filled. To complete these simulations with thousands of macroparticles per bunch, we perform hundreds of simulations simultaneously by using the University of Wisconsin-Madison's CONDOR ${ }^{\circledR}$ pool for highthroughput computing. This pool performs computations by utilizing the otherwise idle time of networked workstations and personal computers [25]. To ensure that sufficiently many macroparticles are used to model broadband impedance, we simulate 4000 macroparticles per bunch. For consistency, we also use 4000 macroparticles per bunch to model a parasitic high- $Q$ HOM, although nearly the same results for a high- $Q$ HOM may be obtained with 60 macroparticles per bunch [26].

\section{B. Parasitic coupled-bunch instability}

When $\omega_{3} / 2 Q_{3}<2 \omega_{0}$, where $\omega_{0}$ is the ring's angular recirculation frequency, the HOM impedance may be excited by a single rotation sideband [27] to cause parasitic coupled-bunch instability. The worst-case scenario is when the HOM resonant frequency equals a sideband frequency. For worst-case instability growth with a parasitic cavity mode of impedance $R_{3}$ and resonant angular frequency $\omega_{3}$, the complex frequency shift is imaginary with magnitude equaling the coupled-bunch growth rate, given by

$$
\left|\Delta \Omega_{C B}\right|=\frac{e I \alpha \omega_{3} R_{3} F_{3}^{2}}{2 E T_{\mathrm{o}} \omega_{R}} .
$$

Here, $F_{3}$ is the bunch form factor at frequency $\omega_{3}$ and $I \geq$ 0 is the ring average current. To approximately model quadratic and nonquadratic synchrotron potentials, $\omega_{R}$ is the calculated frequency of a rigid-dipole oscillation [8], which equals the linear synchrotron frequency for a quadratic synchrotron potential. If the growth rate remains positive after subtracting the radiation-damping rate $\tau_{L}{ }^{-1}$, it is compared with the dipole Landau damping rate [8] to estimate whether Landau damping is sufficient to prevent parasitic coupled-bunch instability. Robinson instabilities are also predicted [8].

Figure 3(a) shows analytic instability modeling for Aladdin operation with the "base" lattice. The base lattice was the standard lattice for $800-\mathrm{MeV}$ operation until 2002;
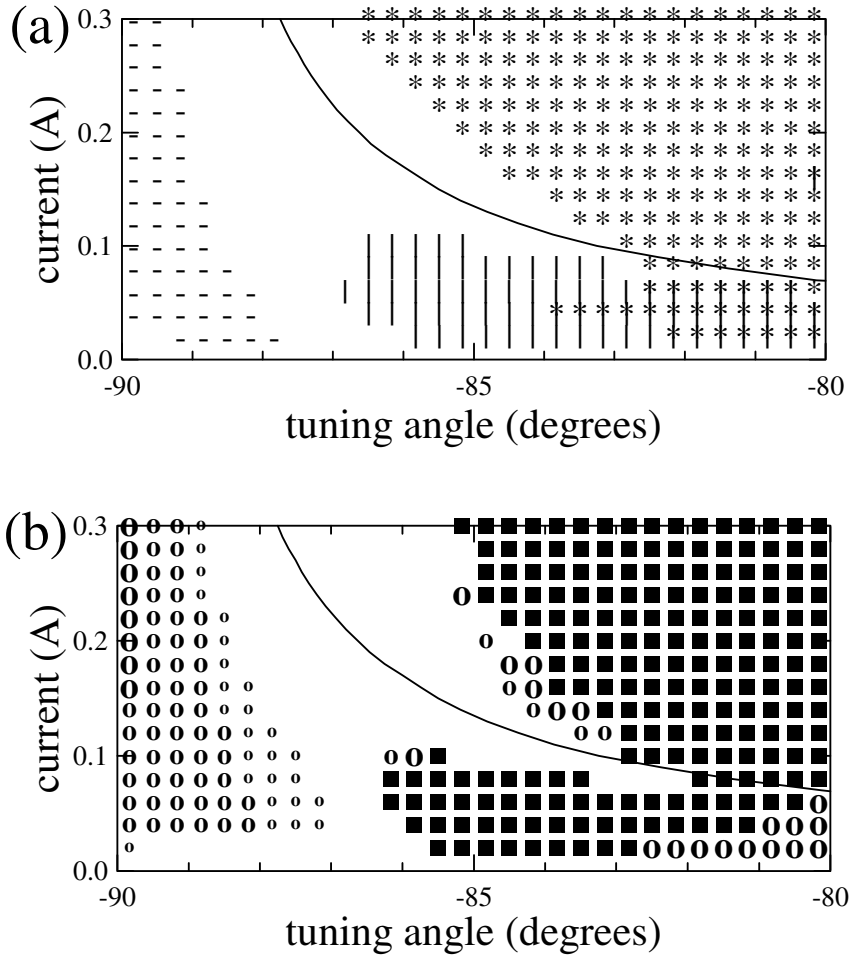

FIG. 3. Modeling for the Aladdin base lattice with worst-case parasitic coupled-bunch instability. A curve shows the parameters for optimal bunch lengthening. (a) Analytic predictions. -: parasitic coupled-bunch instability; I: coupled-dipole Robinson instability; *: coupled-quadrupole Robinson instability. (b) Results of 500000-turn simulations of 60000 macroparticles. $\mathrm{o}$ : mild instability, where the energy spread exceeds its natural value by (10-30)\%; o: moderate instability, where the energy spread has increased by $(30-100) \%$; O: strong instability, where the energy spread has increased more than $100 \%$; $\mathbf{\square}$ : lost macroparticles.

it is now used for $108-\mathrm{MeV}$ injection, ramping to $800 \mathrm{MeV}$, and $1-\mathrm{GeV}$ operation. For this lattice, the passive harmonic cavity is operated with its circulator detached, so that its $\mathrm{rf}$ coupling $\beta_{2}$ equals zero. A variety of ring currents and passive harmonic-cavity tuning angles are considered for an $\mathrm{HOM}$ with $Q_{3}=3000, R_{3}=10 \mathrm{k} \Omega$, and $\omega_{3}=$ $6.28319 \times 10^{9} \mathrm{rad} / \mathrm{s}$. When instability is predicted analytically, a symbol is plotted. A curve is plotted to show the parameters for optimal bunch lengthening of a stable beam - when the quadratic component of the synchrotron effective potential vanishes and the linear synchrotron frequency is zero. Optimal bunch lengthening is calculated to increase the bunch length by a factor of 2.4 , from its natural value of 255 to $600 \mathrm{ps}$.

According to the analytic modeling, Robinson instability will prevent the harmonic cavity from being tuned for stable optimal bunch lengthening at ring currents lower than $100 \mathrm{~mA}$. For higher currents, optimal bunch lengthening is predicted to suppress the parasitic coupled-bunch instability and double the bunch length without exciting Robinson instability. Overstretching the bunches with 
higher harmonic-cavity voltages is predicted to excite the coupled-quadrupole Robinson instability. These predictions agree with experiment [8].

Simulations of 60000 macroparticles (4000 macroparticles per bunch in 15 bunches) circulating for 500000 turns were performed for the same ring currents and tuning angles as the analytic predictions. For each simulation, the relative energy spread $\sigma_{E} / E$ and the number of lost macroparticles at the end of the simulation were recorded. An energy spread that exceeds the natural value by more than $10 \%$ is taken as a sign of instability. In Fig. 3(b), a solid square is plotted when macroparticles are lost in a simulation, while a circle is plotted when the energy spread at the end of a simulation exceeds the natural value by more than $10 \%$. A small circle is plotted for mild instability [when $\sigma_{E} / E$ exceeds the natural value by (10-30)\%]. A mediumsize circle is plotted for moderate instability [when $\sigma_{E} / E$ exceeds the natural value by $(30-100) \%$, while a large circle is plotted for strong instability (when $\sigma_{E} / E$ exceeds the natural value by more than $100 \%$ ). For mild instability, the energy spread resulting from instability is small compared to the natural energy spread, while the energy spread from instability dominates the natural energy spread for a strong instability. Strong instabilities should be avoided at Aladdin, since a large increase in energy spread diminishes the performance of some insertion devices. Macroparticles may be lost from instability or at the beginning of a simulation. In this article, lost macroparticles occur for parameters where instabilities are predicted, consistent with their loss from instability.

There is good agreement between the analytic predictions of Fig. 3(a) and the simulated instabilities observed in Fig. 3(b), confirming that tuning in the harmonic cavity strongly suppresses the parasitic coupled-bunch instability.

In Fig. 4(a), analytic modeling results are shown for the Aladdin low-emittance lattice, whose momentum compaction is much lower than the base lattice. For this lattice, the harmonic cavity is operated with its circulator attached, so that its rf coupling $\beta_{2}$ equals 1.5. A coupled-dipole Robinson instability is predicted to destabilize partially lengthened bunches for ring currents less than $60 \mathrm{~mA}$, while a fast dipole-quadrupole Robinson mode-coupling instability is predicted to destabilize optimally lengthened bunches for higher currents. This agrees with simulations and experiment $[8,28]$. For optimally lengthened bunches, suppression of the parasitic coupled-bunch instability is predicted. Optimal bunch lengthening is calculated to increase the bunch length by a factor of 3.2, from its natural value of 148 to $475 \mathrm{ps}$.

Simulation results for a parasitic HOM are shown in Fig. 4(b). The analytic instability predictions of Fig. 4(a) agree approximately with Fig. 4(b). For ring currents exceeding $100 \mathrm{~mA}$, instabilities may be avoided in simulations by using a double-hump bunch shape obtained with a harmonic-cavity voltage exceeding that required for opti-
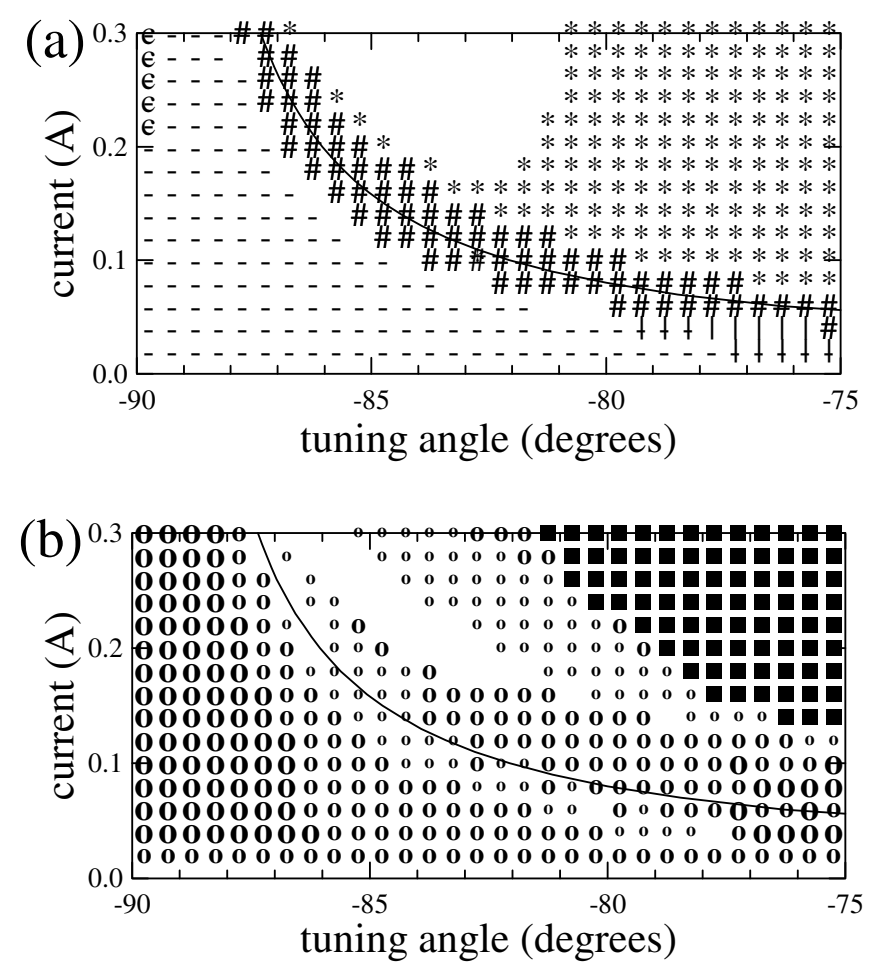

FIG. 4. Modeling for the Aladdin low-emittance lattice with worst-case parasitic coupled-bunch instability. A curve shows parameters for optimal bunch lengthening. (a) Analytic predictions. -: parasitic coupled-bunch instability; I: coupled-dipole Robinson instability; *: coupled-quadrupole Robinson instability; \#: fast dipole-quadrupole mode-coupling Robinson instability; c: coupled-bunch instability with longitudinal mode number of 1. (b) Results of 500000-turn simulations of 60000 macroparticles. o: mild instability, where the energy spread exceeds its natural value by $(10-30) \% ; \mathbf{o}$ : moderate instability, where the energy spread has increased by (30-100)\%; O: strong instability, where the energy spread has increased more than $100 \%$; $\mathbf{\square}$ : lost macroparticles.

mal bunch lengthening by $20 \%$. This agrees with experiment, so that our standard ring operation employs such double-hump bunches to avoid Robinson and parasitic coupled-bunch instabilities throughout the operating current range of 100-300 mA. A comparison of Figs. 3 and 4 indicates that suppression of parasitic coupled-bunch instabilities by the harmonic cavity requires more bunch lengthening in the low-emittance lattice than in the base lattice, which is also in agreement with experiment [8].

Figure 5(a) displays analytic modeling for MAX-II, which predicts suppression of the parasitic coupled-bunch instability by tuning in the harmonic cavity. The coupleddipole Robinson instability is predicted to destabilize partially lengthened bunches for ring currents less than $80 \mathrm{~mA}$, while a fast dipole-quadrupole Robinson modecoupling instability is predicted to destabilize optimally lengthened bunches for currents of 80-240 mA. Optimal bunch lengthening is calculated to increase the bunch 

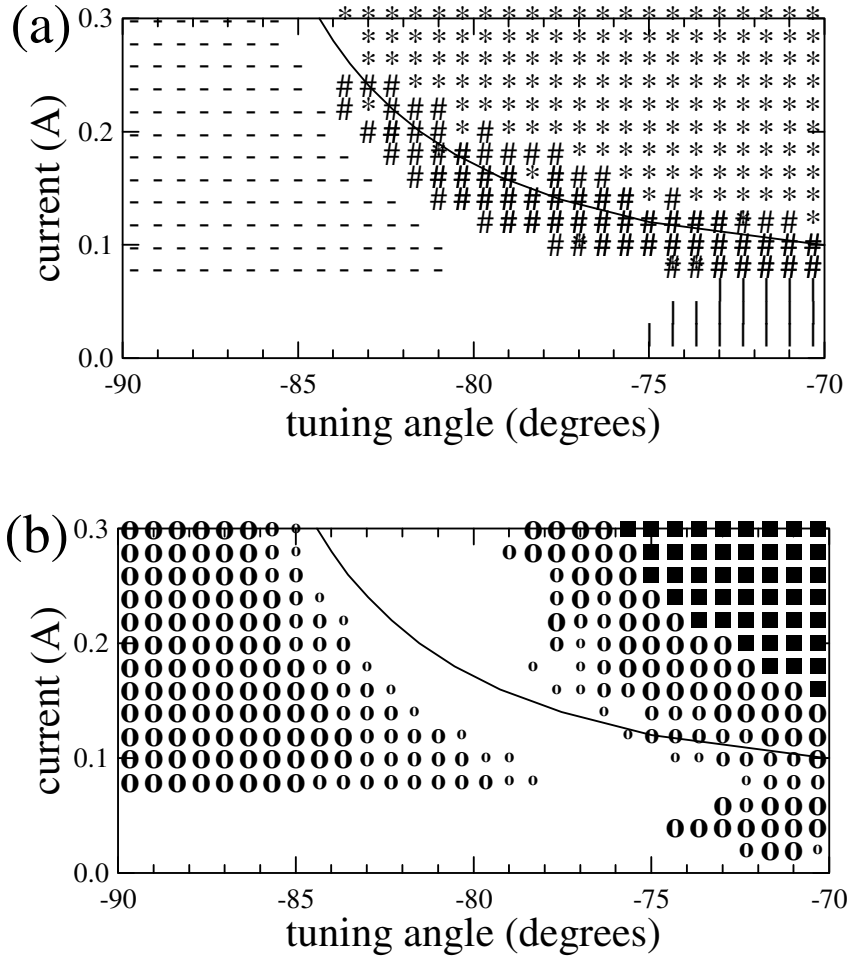

FIG. 5. Modeling for the new MAX-II 100-MHz/500-MHz rf system with worst-case parasitic coupled-bunch instability. A curve shows the parameters for optimal bunch lengthening. (a) Analytic predictions. -: parasitic coupled-bunch instability; I: coupled-dipole Robinson instability; *: coupled-quadrupole Robinson instability; \#: fast dipole-quadrupole mode-coupling Robinson instability. (b) Results of 500 000-turn simulations of 120000 macroparticles. o: mild instability, where the energy spread exceeds its natural value by $(10-30) \%$; o: moderate instability, where the energy spread has increased by (30100)\%; O: strong instability, where the energy spread has increased more than $100 \%$; $\mathbf{\square}$ : lost macroparticles.

length by a factor of 3.5 , from its natural value of 57 to 200 ps.

The analytic Robinson-instability predictions are in approximate agreement with 120000 -macroparticle simulations shown in Fig. 5(b). The analytic predictions for parasitic coupled-bunch instability of Fig. 5(a) are in good agreement with simulations shown in Fig. 5(b). Tuning in the harmonic cavity strongly suppresses the parasitic coupled-bunch instability while increasing the bunch length.

Modeling has also been performed for UVSOR, MAX-II with the previous $500-\mathrm{MHz} / 1500-\mathrm{MHz}$ rf system, MAXIII, and the MAX-IV ring designs for 1.5 and $3 \mathrm{GeV}$ ring energies [26]. In all cases, simulations confirm that parasitic coupled-bunch instabilities are strongly suppressed by tuning in the passive harmonic cavity, in agreement with the analytic model. For the Aladdin and UVSOR rings, the analytic predictions and simulations are further supported by experimental observations of Robinson instabilities and parasitic coupled-bunch instabilities [8-10]. For Aladdin, the loss of macroparticles in simulations is in approximate agreement with experimental observations of beam losses.

\section{Microwave instability}

To estimate whether microwave instability will be caused by a broadband HOM when $\omega_{3} / 2 Q_{3}>2 \omega_{0}$, we consider the negative-mass instability of a uniform coasting beam whose current equals the peak bunch current $I_{\text {peak }}$ [11]. For ring energies large compared to the transition energy, the Keil-Schnell instability criterion for mode frequency $\omega$ gives an approximate threshold peak current [29]

$$
I_{\text {peak }_{\text {trreshold }}}=\frac{E}{e} T_{\mathrm{o}}|\alpha|\left(\frac{\sigma_{E_{\text {nat }}}}{E}\right)^{2}\left|\frac{\omega}{Z(\omega)}\right| .
$$

Here, $\sigma_{E_{\text {nat }}}$ is the beam's natural energy spread and $Z(\omega)$ is the impedance. The threshold current increases with $\omega$ for typical impedance models. We conservatively estimate that the smallest possible mode frequency is $\omega \approx 1 / \sigma_{t}$, as in the ZAP code [12]. For a HOM characterized by $\omega_{3}, R_{3}$, and $Q_{3}$, the threshold peak current is therefore given by letting $\omega=1 / \sigma_{t}$ in Eq. (4). For a Gaussian bunch shape [where $I_{\text {peak }}=(2 \pi)^{1 / 2} I / \omega_{g} \sigma_{t}$ ], the threshold ring current is

$$
\begin{aligned}
I_{\text {threshold }}= & \frac{\omega_{g}}{\sqrt{2 \pi}} \frac{E}{e} T_{\mathrm{o}}|\alpha|\left(\frac{\sigma_{E_{\text {nat }}}}{E}\right)^{2} \\
& \times \frac{\left[1+Q_{3}{ }^{2}\left(\omega_{3} \sigma_{t}-1 / \omega_{3} \sigma_{t}\right)^{2}\right]^{1 / 2}}{R_{3}} .
\end{aligned}
$$

In computing the threshold current, we compute $\sigma_{t}$ without considering any potential-well distortion from the broadband HOM. Since Eq. (5) was obtained by considering a uniform coasting beam and estimating the smallest possible mode frequency, we expect that our analytic estimate of microwave instability thresholds will be approximate.

In the long-bunch regime $\left(\omega_{3} \sigma_{t}>1\right)$, Eq. (5) indicates that the threshold current increases with increasing bunch length, because increasing the bunch length decreases the peak current. In the short-bunch regime $\left(\omega_{3} \sigma_{t}<1\right)$, the threshold current decreases with increasing bunch length, because the dependence of the impedance upon the mode frequency $\omega \approx 1 / \sigma_{t}$ has a greater effect upon the threshold than the peak current. Therefore, lengthening the bunch with a harmonic cavity is expected to suppress microwave instability in the long-bunch regime, as observed in Refs. $[3,13]$. In the short-bunch regime, increasing the bunch length with a harmonic cavity is expected to worsen microwave instability.

At Aladdin, the microwave instability may be driven by the reduced longitudinal broadband impedance of the vacuum chamber, estimated to be $\left|Z_{p} / p\right| \approx 5.7 \Omega$ with cutoff angular frequency $\omega_{c}=8 \times 10^{9} \mathrm{rad} / \mathrm{s}$ [18]. We model this impedance by a HOM with $\omega_{3}=\omega_{c}=8 \times$ $10^{9} \mathrm{rad} / \mathrm{s}, Q_{3}=1$ and $R_{3}=2148 \Omega$, since a broadband 
resonator model is able to reproduce all of the microwave instability-related phenomena [13].

In Fig. 6, the microwave instability is considered for the Aladdin base lattice. For the natural bunch length of $255 \mathrm{ps}, \omega_{3} \sigma_{t}=2$, so that tuning in the harmonic cavity is expected to suppress the microwave instability. In Fig. 6(a), an " $m$ " is plotted when the microwave instability is predicted analytically for the Aladdin base lattice. Robinson-instability predictions are also shown. The results of 60000 -macroparticle simulations are shown in Fig. 6(b). Approximate agreement between the analytic predictions and simulations is obtained. The simulations confirm the analytic prediction that microwave instability occurs at high ring currents with the harmonic cavity detuned, and is suppressed by lengthening the bunch with the harmonic cavity.

Figure 7 shows modeling of the same broadband impedance with the Aladdin low-emittance lattice. For the natural bunch length of $148 \mathrm{ps}, \omega_{3} \sigma_{t}=1.2$, which is slightly within the long-bunch regime. Tuning in the harmonic
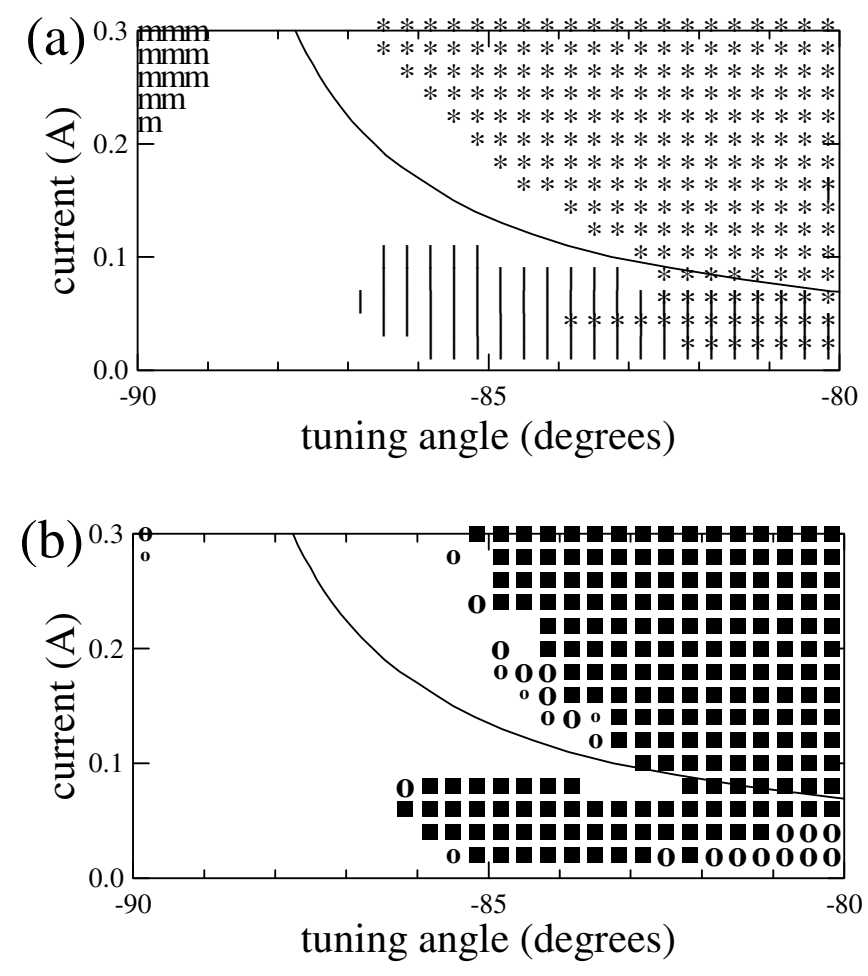

FIG. 6. Modeling for the Aladdin base lattice with microwave instability from the broadband impedance $\left|Z_{p} / p\right|=5.7 \Omega$. A solid curve shows the parameters for optimal bunch lengthening. (a) Analytic predictions. m: microwave instability; I: coupleddipole Robinson instability; *: coupled-quadrupole Robinson instability. (b) Results of 500000 -turn simulations of 60000 macroparticles. o: mild instability, where the energy spread exceeds its natural value by $(10-30) \%$; $\mathbf{o}$ : moderate instability, where the energy spread has increased by (30-100)\%; O: strong instability, where the energy spread has increased more than $100 \%$; $\mathbf{\square}$ : lost macroparticles. cavity is therefore expected to suppress the microwave instability. The occurrence of microwave instability in simulations is approximately described by the analytic predictions. With the harmonic cavity detuned, the microwave instability is observed in simulations when the ring current exceeds $60 \mathrm{~mA}$. When the harmonic cavity lengthens the bunch to its standard double-hump shape, the microwave instability occurs in simulations when the ring current exceeds $180 \mathrm{~mA}$. The simulated instability thresholds agree with experiment [8], confirming that use of the harmonic cavity increases the microwave instability threshold by a factor of 3 .

The increased energy spread for standard operation with currents exceeding $180 \mathrm{~mA}$ enlarges the beam's horizontal size, which is disadvantageous for some ring users. Simulations shown in Fig. 8 predict that decreasing the ring's broadband impedance by one-third (to $\left|Z_{p} / p\right|=$ $3.8 \Omega$ ) would prevent microwave instability for standard
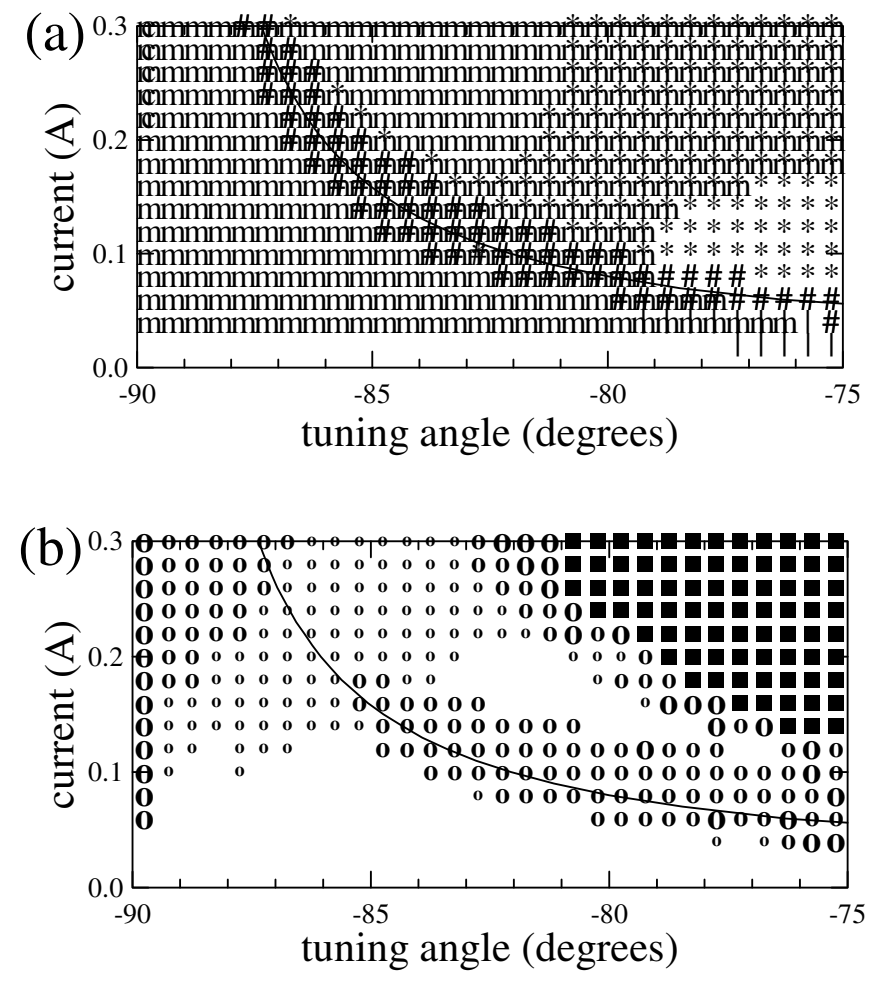

FIG. 7. Modeling for the Aladdin low-emittance lattice with microwave instability from the broadband impedance $\left|Z_{p} / p\right|=$ $5.7 \Omega$. A curve shows the parameters for optimal bunch lengthening. (a) Analytic predictions. m: microwave instability; I: coupled-dipole Robinson instability; *: coupled-quadrupole Robinson instability; \#: fast dipole-quadrupole mode-coupling Robinson instability; c: coupled-bunch instability with longitudinal mode number of 1. (b) Results of 500000 -turn simulations of 60000 macroparticles. o: mild instability, where the energy spread exceeds its natural value by $(10-30) \%$; o: moderate instability, where the energy spread has increased by $(30-$ 100)\%; O: strong instability, where the energy spread has increased more than 100\%; $\mathbf{\square}$ : lost macroparticles. 

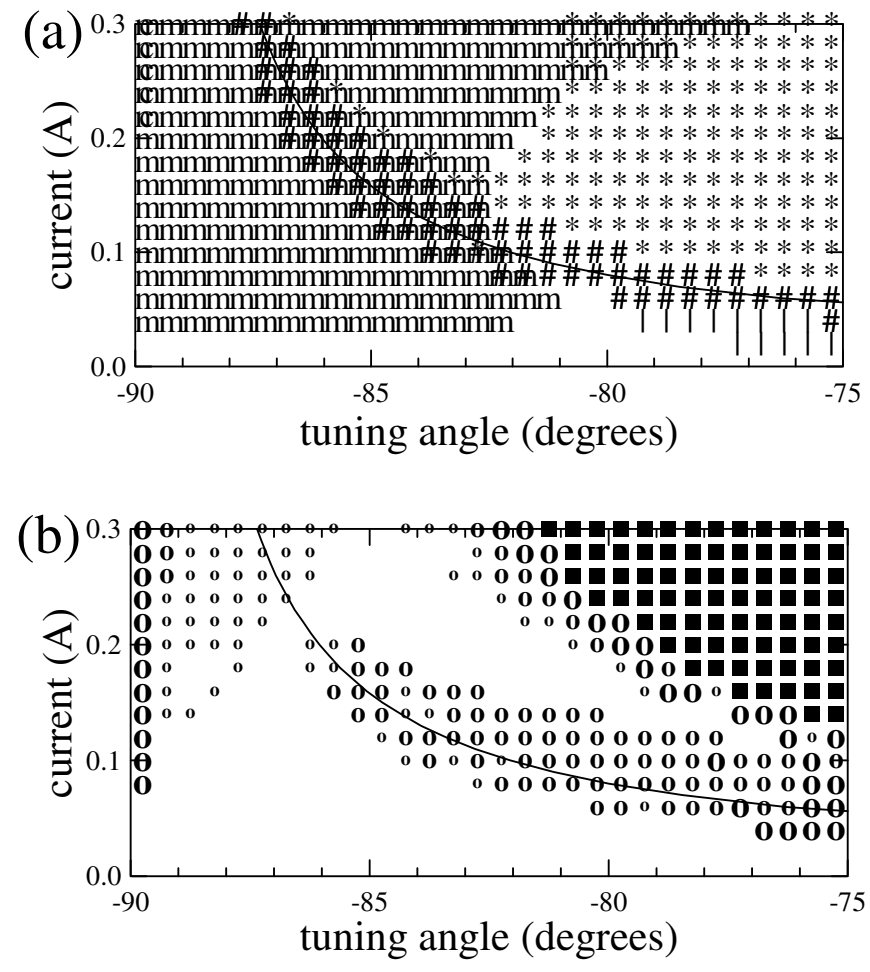

FIG. 8. Modeling for the Aladdin low-emittance lattice with microwave instability from the broadband impedance $\left|Z_{p} / p\right|=$ $3.8 \Omega$. A curve shows the parameters for optimal bunch lengthening. (a) Analytic predictions. m: microwave instability; I: coupled-dipole Robinson instability; *: coupled-quadrupole Robinson instability; \#: fast dipole-quadrupole mode-coupling Robinson instability; c: coupled-bunch instability with longitudinal mode number of 1 . (b) Results of 500 000-turn simulations of 60000 macroparticles. o: mild instability, where the energy spread exceeds its natural value by $(10-30) \%$; o: moderate instability, where the energy spread has increased by (30100)\%; O: strong instability, where the energy spread has increased more than 100\%; $\mathbf{\square}$ : lost macroparticles.

operation throughout our operating current range of $100-$ $300 \mathrm{~mA}$. Modifying the ring's beam position monitors, ionclearing electrodes and/or bellows may give the desired impedance reduction [18].

The MAX-II vacuum-chamber dimensions are comparable to Aladdin. Both chambers contain unshielded bellows, untapered beam pipe transitions and a $Q$ electrode. However, the MAX-II chamber does not contain strip line beam position monitors that contribute a substantial portion of Aladdin's calculated broadband impedance. Thus, the estimated Aladdin reduced broadband impedance of $5.7 \Omega$ provides a pessimistic estimate for MAX-II. To model $\left|Z_{p} / p\right|=5.7 \Omega$ for MAX-II, we consider HOM parameters $Q_{3}=1, \quad \omega_{3}=8 \times 10^{9} \mathrm{rad} / \mathrm{s}, \quad$ and $R_{3}=$ $2177 \Omega$. For the natural bunch length of $57 \mathrm{ps}, \omega_{3} \sigma_{t}=$ 0.5 , so that the natural bunch length is within the shortbunch regime. Tuning in the harmonic cavity is predicted to worsen the microwave instability until the bunch length is doubled. Further tuning in the harmonic cavity gives a bunch length in the long-bunch regime, so that additional bunch lengthening is expected to suppress the microwave instability.

Analytic predictions and results of 120000 macroparticle simulations are shown in Fig. 9. Approximate agreement is obtained. As expected, tuning in the harmonic cavity initially worsens the microwave instability. Additional bunch lengthening suppresses the microwave instability, so that microwave instability does not occur in simulations of optimal bunch lengthening. Therefore, the microwave instability is not expected for MAX-II operation with optimally lengthened bunches.

For the cases studied, the threshold current for microwave instability is lower in the analytic model than in the simulations by as much as a factor of 2 . This discrepancy is partially because of our criterion that the energy spread in a simulation must increase by more than $10 \%$ to indicate
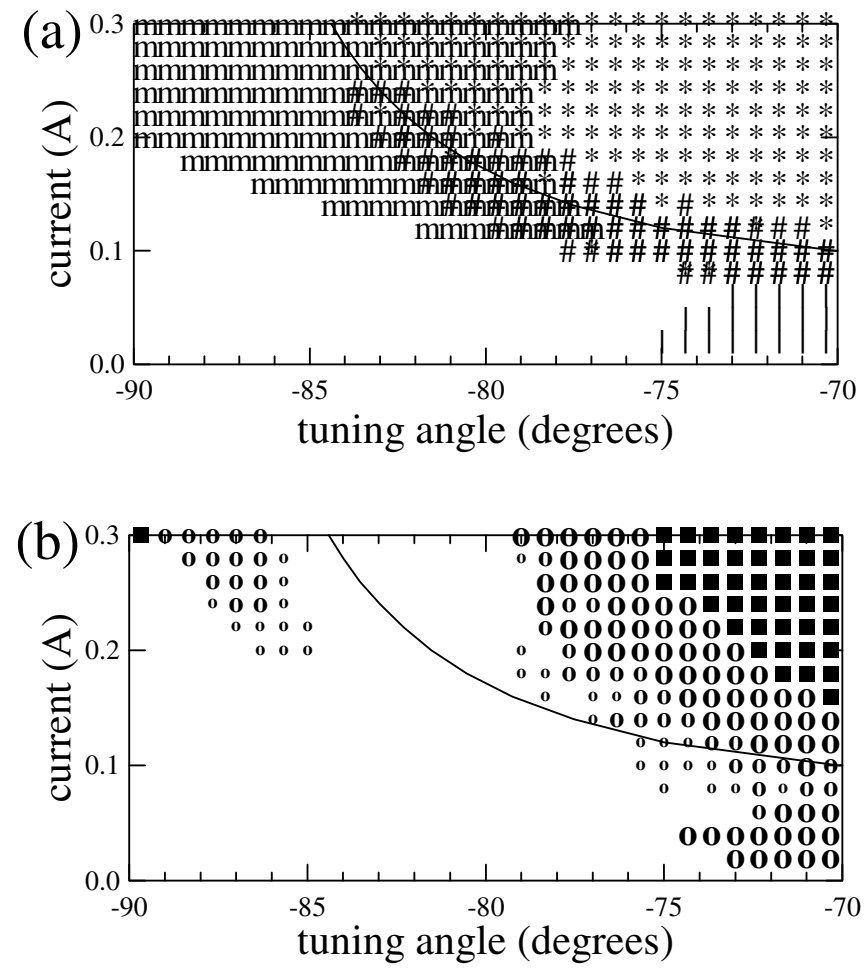

FIG. 9. Modeling for the new MAX-II 100-MHz/500-MHz rf system, with microwave instability from the broadband impedance $\left|Z_{p} / p\right|=5.7 \Omega$. A solid curve shows the parameters for optimal bunch lengthening. (a) Analytic predictions. m: microwave instability; I: coupled-dipole Robinson instability; *: coupled-quadrupole Robinson instability; \#: fast dipolequadrupole mode-coupling Robinson instability. (b) Results of 500000-turn simulations of 120000 macroparticles. o: mild instability, where the energy spread exceeds its natural value by $(10-30) \%$; $\mathbf{o}$ : moderate instability, where the energy spread has increased by $(30-100) \%$; O: strong instability, where the energy spread has increased more than 100\%; $\mathbf{\square}$ : lost macroparticles. 
instability - requiring a ring current $20 \%$ above threshold according to Eq. (5). The majority of the discrepancy may be attributed to the approximate nature of the analytic coasting-beam model. Microwave instability thresholds in simulations more accurately describe Aladdin experimental results than the analytic model.

\section{SUMMARY}

The effect of a passive harmonic cavity upon longitudinal stability has been studied for the Aladdin electron storage ring operated in base-lattice or low-emittance tunings, and for the MAX-II electron storage ring. According to analytic modeling and simulations, parasitic coupledbunch instabilities excited by a typical HOM are strongly suppressed by tuning in the harmonic cavity. Experiments at Aladdin further confirm the suppression of parasitic coupled-bunch instabilities.

At Aladdin, the natural bunch length exceeds the vacuum-chamber radius. In this long-bunch regime of the microwave instability, analytic modeling and simulations indicate that tuning in the harmonic cavity suppresses microwave instability. For low-emittance operation, the microwave instability thresholds in experiments are in good agreement with simulations, confirming that tuning in the harmonic cavity increases the threshold current by a factor of 3, to $180 \mathrm{~mA}$. According to simulations with the harmonic cavity tuned in, reducing the ring's broadband impedance by one-third would increase the threshold current for microwave instability to the maximum operating ring current of $300 \mathrm{~mA}$.

For MAX-II, the natural bunch length is smaller than the vacuum-chamber radius. In this short-bunch regime of the microwave instability, analytic modeling and simulations predict that tuning in the harmonic cavity initially worsens the microwave instability. When the bunch is lengthened sufficiently, so that it is in the long-bunch regime of the microwave instability, additional bunch lengthening suppresses the microwave instability. Consequently, the microwave instability is not expected for MAX-II when the bunches are optimally lengthened by the harmonic cavity.

For the cases studied, tuning in a passive harmonic cavity strongly suppresses parasitic coupled-bunch instabilities from a high- $Q$ higher-order mode. When the natural bunch length is within the long-bunch regime of the microwave instability, tuning in a passive harmonic cavity also suppresses the microwave instability.

\section{ACKNOWLEDGMENTS}

The author thanks $\AA$. Andersson and M. Eriksson for MAX-II parameters, P. J. Hagen, M. A. Green, J. P. Stott, and K.D. Jacobs for computational assistance, K. J. Kleman and J.J. Bisognano for valuable discussions. Computations were performed on the Condor ${ }^{\circledR}$ highthroughput computing pool of the Computer Sciences
Department, University of Wisconsin-Madison. This work is based upon research conducted at the Synchrotron Radiation Center, University of WisconsinMadison, which is supported by the National Science Foundation (Grant No. DMR-0084402). The experimental data used in Fig. 2 are courtesy of K. D. Jacobs and K. J. Kleman.

\section{APPENDIX: COMPUTATIONAL METHODS}

\section{A. Wake fields}

When a particle of charge $q<0$ passes through an rf cavity at time $t=0$, the physical wake field at $t>0$ from a cavity mode with resonant angular frequency $\omega$ is [27]

$$
\Delta V(t)=q(2 R / \tau) e^{-t / \tau}[\cos \bar{\omega} t-(1 / \bar{\omega} \tau) \sin \bar{\omega} t]
$$

where $R$ is the impedance at resonance, $Q>\frac{1}{2}$ is the quality factor, $\tau \equiv 2 Q / \omega$ is the mode's damping time, and $\bar{\omega} \equiv$ $\omega \sqrt{1-(1 / 2 Q)^{2}}$. The physical wake field equals 0 for $t<$ 0 and one-half the value given by Eq. (A1) for $t=0$. In our computations, we consider an "analytic" wake field given by Eq. (A1) for $-\infty<t<\infty$, so that a particle's wake contributes to the cavity voltage at all times, including times which precede the particle's passage through the cavity. This analytic wake field may be used to compute forces at times after the particle passes through the cavity, while one-half of the analytic wake at $t=0$ gives the effect of a particle's wake upon itself.

Consider the superposition of analytic wake fields from many macroparticles passing through an rf cavity many times. Let $t_{g}$ equal the $\mathrm{rf}$ period $2 \pi / \omega_{g}$, and let $t=0$ correspond to a stable synchronous phase of the steadystate rf voltage. For any non-negative integer $\nu$, we may represent this superposition by in-phase and quadrature components $V_{c}(t)$ and $V_{s}(t)$ [17] relative to the harmonic frequency $\nu \omega_{g}$

$$
V(t)=V_{c}(t) \cos \left(\nu \omega_{g} t\right)-V_{s}(t) \sin \left(\nu \omega_{g} t\right)
$$

Equation (A1) indicates that the analytic wake of a particle passing through the rf cavity at time $t_{n}$ modifies these components by

$$
\begin{aligned}
\Delta V_{c}(t)= & q(2 R / \tau) e^{-\left(t-t_{n}\right) / \tau}\left[\cos \left(\Delta \omega t-\bar{\omega} t_{n}\right)-(1 / \bar{\omega} \tau)\right. \\
& \left.\times \sin \left(\Delta \omega t-\bar{\omega} t_{n}\right)\right], \\
\Delta V_{s}(t)= & q(2 R / \tau) e^{-\left(t-t_{n}\right) / \tau}\left[\sin \left(\Delta \omega t-\bar{\omega} t_{n}\right)+(1 / \bar{\omega} \tau)\right. \\
& \left.\times \cos \left(\Delta \omega t-\bar{\omega} t_{n}\right)\right],
\end{aligned}
$$

for $-\infty<t<\infty$, where $\Delta \omega \equiv \bar{\omega}-\nu \omega_{g}$. For a superposition of analytic wakes from numerous macroparticles, the components change in time according to 


$$
\begin{aligned}
& V_{c}\left[t+t_{1}\right]=e^{-t_{1} / \tau}\left[V_{c}(t) \cos \Delta \omega t_{1}-V_{s}(t) \sin \Delta \omega t_{1}\right], \\
& V_{s}\left[t+t_{1}\right]=e^{-t_{1} / \tau}\left[V_{s}(t) \cos \Delta \omega t_{1}+V_{c}(t) \sin \Delta \omega t_{1}\right] .
\end{aligned}
$$

For integral $n$, the analytic wake produced at synchronous time $t=n t_{g}$ by a particle passing through the cavity at time $n t_{g}+t_{m}$ is given by Eq. (A3) as

$$
\begin{aligned}
& \Delta V_{c}\left(n t_{g}\right)=q(2 R / \tau) e^{t_{m} / \tau}\left[\cos \bar{\omega} t_{m}+(1 / \bar{\omega} \tau) \sin \bar{\omega} t_{m}\right], \\
& \Delta V_{s}\left(n t_{g}\right)=-q(2 R / \tau) e^{t_{m} / \tau}\left[\sin \bar{\omega} t_{m}-(1 / \bar{\omega} \tau) \cos \bar{\omega} t_{m}\right] .
\end{aligned}
$$

From Eq. (A4), the cavity field experienced by a macroparticle passing through the cavity at time $n t_{g}+t_{m}$ is related to the components at the synchronous time $n t_{g}$ by

$$
\begin{aligned}
V\left(n t_{g}+t_{m}\right)= & e^{-t_{m} / \tau}\left[V_{c}\left(n t_{g}\right) \cos \Delta \omega t_{m}\right. \\
& \left.-V_{s}\left(n t_{g}\right) \sin \Delta \omega t_{m}\right] \cos \left(\nu \omega_{g} t_{m}\right) \\
& -e^{-t_{m} / \tau}\left[V_{s}\left(n t_{g}\right) \cos \Delta \omega t_{m}\right. \\
& \left.+V_{c}\left(n t_{g}\right) \sin \Delta \omega t_{m}\right] \sin \left(\nu \omega_{g} t_{m}\right) .
\end{aligned}
$$

The fields at synchronous time $(n+1) t_{g}$ are also given by Eq. (A4)

$$
\begin{aligned}
V_{c}\left[(n+1) t_{g}\right]= & e^{-t_{g} / \tau}\left[V_{c}\left(n t_{g}\right) \cos \Delta \omega t_{g}-V_{s}\left(n t_{g}\right)\right. \\
& \left.\times \sin \Delta \omega t_{g}\right], \\
V_{s}\left[(n+1) t_{g}\right]= & e^{-t_{g} / \tau\left[V_{s}\left(n t_{g}\right) \cos \Delta \omega t_{g}+V_{c}\left(n t_{g}\right)\right.} \\
& \left.\times \sin \Delta \omega t_{g}\right] .
\end{aligned}
$$

\section{B. Dominant modes of the rf cavities}

Consider the high- $Q$ dominant mode of a rf cavity whose resonant angular frequency $\omega$ is near to $\nu \omega_{g}$ for a positive integer $\nu$. For Aladdin, $\nu=1$ describes the fundamental rf cavity while $\nu=4$ describes the harmonic rf cavity. We describe the wake fields using the in-phase and quadrature components relative to the frequency $\nu \omega_{g}$, where computations are speeded by approximating Eqs. (A5) and (A6) as

$$
\begin{aligned}
& \Delta V_{c}\left(n t_{g}\right)=q\left[(2 R / \tau) \cos \nu \omega_{g} t_{m}+(R / Q \tau) \sin \nu \omega_{g} t_{m}\right], \\
& \Delta V_{s}\left(n t_{g}\right)=q\left[(R / Q \tau) \cos \nu \omega_{g} t_{m}-(2 R / \tau) \sin \nu \omega_{g} t_{m}\right],
\end{aligned}
$$

and

$V\left(n t_{g}+t_{m}\right)=V_{c}\left(n t_{g}\right) \cos \left(\nu \omega_{g} t_{m}\right)-V_{s}\left(n t_{g}\right) \sin \left(\nu \omega_{g} t_{m}\right)$.

Tracking with Eqs. (A7)-(A9) is described in Eqs. (23)(31) of Ref. [8].

For steady-state operation, the wake field components relative to the frequency $\nu \omega_{g}$ are constant, given by [8]

$$
V_{c}=-2 F I R \cos ^{2} \phi, \quad V_{s}=F I R \sin 2 \phi,
$$

where $F$ is the bunch form factor for frequency $\nu \omega_{g}$, and $\phi$ is the cavity's tuning angle, defined by $\tan \phi \equiv 2 Q\left(\nu \omega_{g}-\right.$ $\omega) / \omega$.

\section{Higher-order modes}

To model a HOM with $Q>1 / 2$, we use the in-phase and quadrature components relative to the harmonic with $\nu=0$, which is equivalent to the method in Ref. [30]. The cavity field equals the in-phase component, while the quadrature component is used to relate fields at different times. Eqs. (A6) and (A7) become

$$
\begin{aligned}
V\left(n t_{g}+t_{m}\right)= & e^{-t_{m} / \tau}\left[V_{c}\left(n t_{g}\right) \cos \left(\bar{\omega} t_{m}\right)-V_{s}\left(n t_{g}\right)\right. \\
& \left.\times \sin \left(\bar{\omega} t_{m}\right)\right],
\end{aligned}
$$

and

$$
\begin{aligned}
& V_{c}\left[(n+1) t_{g}\right]=e^{-t_{g} / \tau}\left[V_{c}\left(n t_{g}\right) \cos \bar{\omega} t_{g}-V_{s}\left(n t_{g}\right) \sin \bar{\omega} t_{g}\right] \\
& V_{s}\left[(n+1) t_{g}\right]=e^{-t_{g} / \tau}\left[V_{s}\left(n t_{g}\right) \cos \bar{\omega} t_{g}+V_{c}\left(n t_{g}\right) \sin \bar{\omega} t_{g}\right] .
\end{aligned}
$$

Equations (A5), (A11), and (A12) are applied to tracking a HOM in the same way that Eqs. (A7)-(A9) are used for the dominant cavity modes.

\section{Additional code features}

Our code can model cavities in which the rf generator power is supplied with a nonzero load angle $\theta_{\mathrm{v} 1}$, where $\theta_{\mathrm{v} 1}$ is positive when the generator current lags the total $\mathrm{rf}$ voltage [9]. This is achieved by generalizing Eq. (21) of Ref. [8], which determines the tuning angle of the fundamental cavity $\phi_{1}$, to obtain [31]

$$
\begin{aligned}
\phi_{1}= & \tan ^{-1}\left[2 F_{1} I R_{1} \sin \psi_{1} / V_{T 1}-\tan \theta_{\mathrm{v} 1}(1\right. \\
& \left.\left.+2 F_{1} I R_{1} \cos \psi_{1} / V_{T 1}\right)\right] .
\end{aligned}
$$

In our code, a macroparticle with $\left|t_{m}\right| \geq t_{g} / 2$ is considered lost, and its wake no longer contributes to the computed fields. To reduce the loss of macroparticles at the beginning of simulations, the initial values of the rf wakefields equal their steady-state values for the dominant $\mathrm{rf}$ modes of the fundamental and harmonic cavities, given by Eq. (A10).

The macroparticles, initially located at the synchronous phase with no energy spread, are tracked for two radiationdamping times using the calculated steady-state rf fields of the dominant rf cavity modes of the fundamental and harmonic cavities. This creates a macroparticle distribution that approximates the steady state. At this point, the simulation begins with the excitation of wakefields by macroparticles and their decay from finite quality factors. The 
HOM wakefields are also included at this point, with the HOM impedance ramped linearly from zero over the first two radiation-damping times of the simulation.

[1] A. Hofmann and S. Myers, in Proceedings of the 11th International Conference on High Energy Accelerators, Geneva (Birkhäuser Verlag, Basel, 1980), p. 610.

[2] E. Weihreter, A. Gaupp, H. G. Hoberg, W.-D. Klotz, P. Kuske, H. Lehr, R. Maier and G. Mülhaupt, IEEE Trans. Nucl. Sci. 32, 2317 (1985).

[3] S. Krinsky and J. M. Wang, Part. Accel. 17, 109 (1985).

[4] K. J. Kleman, in Proceedings of the 1995 Particle Accelerator Conference, Dallas (IEEE, Piscataway, NJ, 1996), p. 1785.

[5] M. Georgsson, A. Andersson and M. Eriksson, Nucl. Instrum. Methods Phys. Res., Sect. A 416, 465 (1998).

[6] S. L. Kramer and J.-M. Wang, Nucl. Instrum. Methods Phys. Res., Sect. A 423, 260 (1999).

[7] J. M. Byrd, S. De Santis, J. Jacob, and V. Serriere, Phys. Rev. ST Accel. Beams 5, 092001 (2002).

[8] R. A. Bosch, K. J. Kleman, and J. J. Bisognano, Phys. Rev. ST Accel. Beams 4, 074401 (2001).

[9] R. A. Bosch, Synchrotron Radiation Center Technical Note No. SRC-205, 2003.

[10] M. Hosaka, M. Katoh, A. Mochihashi, J. Yamazaki, K. Hayashi, and T. Kinoshita, in Proceedings of the 25th ICFA Advanced Beam Dynamics Workshop: Shanghai Symposium on Intermediate-Energy Light Sources, Shanghai, 2001, edited by J. Corbett, Z. M. Dai, D. Einfeld, and Z. T. Zhao (SSRC, Shanghai, 2002), pp. 171-173.

[11] D. Boussard, CERN Laboratory Report No. LabII-rf-Int.75-2, 1975.

[12] M.S. Zisman, S. Chattopadhyay, and J. J. Bisognano, Lawrence Berkeley Laboratory Report No. LBL-21270, 1986.

[13] A. Mosnier, Nucl. Instrum. Methods Phys. Res., Sect. A 438, 225 (1999).

[14] N. Towne, Phys. Rev. ST Accel. Beams 4, 114401 (2001).

[15] V. Serrière and J. Jacob, in Proceedings of the 8th European Particle Accelerator Conference, Paris (EPSIGA and CERN, Geneva, 2002), p. 748.
[16] M.H. Wang and A. Chao, in Proceedings of the 9th European Particle Accelerator Conference, Lucerne, Switzerland (EPS-AG, Geneva, 2004), p. 2140.

[17] S. Haykin, Communication Systems (Wiley, New York, 1994).

[18] R. A. Bosch and M. V. Fisher, Synchrotron Radiation Center Technical Note No. SRC-207, 2004.

[19] J. J. Bisognano, R. A. Bosch, D. E. Eisert, M. A. Green, K. J. Kleman, and W. S. Trzeciak, in Proceedings of the 2001 Particle Accelerator Conference, Chicago (IEEE, Piscataway, NJ, 2001), p. 2671.

[20] A Andersson, M. Bergqvist, M. Eriksson, L. Malmgren, and L. Thånell, in Proceedings of the 8th European Particle Accelerator Conference, Paris (EPS-IGA and CERN, Geneva, 2002), p. 2118.

[21] Å Andersson, M. Bergqvist, M. Brandin, M. Eriksson, L. Malmgren, and H. Tarawneh, in Proceedings of the 9th European Particle Accelerator Conference, Lucerne, Switzerland (EPS-AG, Geneva, 2004), p. 2158.

[22] P. B. Wilson, K. L.F. Bane, and K. Satoh, IEEE Trans. Nucl. Sci. 28, 2525 (1981).

[23] R. Siemann, Nucl. Instrum. Methods Phys. Res. 203, 57 (1982).

[24] K. L. F. Bane and K. Oide, in Proceedings of the 1993 Particle Accelerator Conference, Washington, DC (IEEE, Piscataway, NJ, 1993), p. 3339.

[25] Condor Team, University of Wisconsin-Madison, Condor Version 6.6.7 Manual (Computer Sciences Department, University of Wisconsin-Madison, Madison, WI, 2004).

[26] R. A. Bosch, Synchrotron Radiation Center Technical Note No. SRC-208, 2004.

[27] A. W. Chao, Physics of Collective Beam Instabilities in High Energy Accelerators (Wiley, NY, 1993).

[28] R. A. Bosch, K. J. Kleman, and J. J. Bisognano, in Proceedings of the 2003 IEEE Particle Accelerator Conference, Portland, OR (IEEE, Piscataway, NJ, 2003), p. 3147.

[29] J.-L. Laclare, in CERN Report No. CERN-85-19, edited by P. Bryant and S. Turner, 1985, p. 377.

[30] C.-Y. Tan and James Steimel, Fermilab Report No. FERMILAB-TM-2163, 2001.

[31] M. Sands, Institut National de Physique Nucleaire et de Physique des Particules, Technical Report No. 2-76, 1976; Eq. (41). 